\title{
A Korean Patient with Kniest Syndrome associated with Lipomeningomyelocele
}

\author{
Hye Won Min ${ }^{1,2}$, Kyo Yeon $\mathrm{Koo}^{1,2}$, Chul Ho Lee', Jeong Yoon Yang and Jin-Sung Lee ${ }^{1,2 *}$ \\ Department of Clinical Genetics ${ }^{1}$, Yonsei University College of Medicine, Seoul, Korea \\ Department of Pediatrics ${ }^{2}$, Yonsei University College of Medicine, Seoul, Korea
}

\begin{abstract}
Kniest syndrome (OMIM \#156550) is a rare autosomal dominant disorder caused by a dysfunction of type II collagen, which is encoded by the COL2A1 gene (OMIM +120140) mapped to chromosome 12q13.11. Type II collagen, a molecule found mostly in the cartilage and vitreous tissues, is essential for the normal development of bones and other connective tissues. Kniest syndrome is a type II collagenopathy that presents as skeletal abnormality associated with disproportionate dwarfism, kyphoscoliosis, enlarged joints, visual loss, hearing loss, and cleft palate. This report describes a Korean patient with Kniest syndrome who was diagnosed with typical clinical features and radiologic findings. The patient presented with disproportionately short stature and kyphoscoliosis from birth. A skeletal survey revealed fused lamina in the thoracic spine, hemivertebrae, flexion deformities in multiple joints, and plagiocephaly.
\end{abstract}

Key words: Kniest syndrome, Collagenopathy, Kyphoscoliosis

\section{Introductions}

Kniest syndrome was originally described as a variant of metatropic dwarfism in 1952 by Wilhelm, ${ }_{1}^{1)}$ but it has been more generally recognized as a distinct entity only in recent years. ${ }^{2)}$ It is a very rare condition, affecting approximately 1 in 1,000,000 live births worldwide, with no sexual predominance. ${ }^{3)}$ Most reported patients are sporadic cases, but the disorder can also be inherited in an autosomal dominant pattern. Kniest syndrome represents one of a spectrum of chondrodysplasias arising from defects in the COL2A1 gene for type II collagen, mapped to 12q13.11.) Type II collagen is an important component of different connective tissues, including cartilage and vitreous tissues, and is essential for the normal development of bones and other connective tissues. ${ }^{5}$ Type II collagenopathies caused by mutations in the COL2A1 gene induce type II achondrogenesis-hydrochondrogenesis, spondyloepiphyseal dysplasia congenita, and Stickler syndrome, along with Kniest syndrome. ${ }^{4,6-8)}$

The clinical features of Kniest syndrome include a disproportionately short stature with a short, barrel-shaped chest, kyphoscoliosis, enlargement of joints with limited range of motion, a flat face with prominent eyes, low nasal bridge, myopia that may progress to retinal detachment, vitreoretinal degeneration, and cleft palate with frequent ear infection. Radiologic findings include short limbs often with bowing, broad metaphyses, dumbbell-shaped femurs, hypoplastic pelvic bones, platyspondyly, vertical clefts of the vertebrae, irregular and large epiphyses with late ossification, diffuse osteoporosis, cloud-like radio-densities, and narrow inter-vertebral disk spaces. Inguinal and umbilical hernias, hearing loss, recurrent

Received: 13 Nov 2012, Revised: 21 Dec 2012, Accepted: 22 Dec 2012, Published: 31 Dec 2012

${ }^{*}$ Corresponding author: Jin-Sung Lee, M.D., D.M.Sc.

Department of Clinical Genetics, Yonsei University College of Medicine, 250 Sungsan-ro, Seodaemun-gu, Seoul 120-752, Korea

Tel: +82-2-2228-2540, Fax: +82-2-362-0755, E-mail: jinsunglee@yuhs.ac

(c) This is an open-access article distributed under the terms of the Creative Commons Attribution Non-Commercial License (http://creativecommons.org/licenses/by-nc/3.0/) which permits unrestricted non-commercial use, distribution, and reproduction in any medium, provided the original work is properly cited.

(c) Copyright 2012 by the Korean Society of Medical Genetics 
otitis media, cataracts, lens dislocation, myopia, visual loss due to retinal detachment, and cleft palate may sometimes be present. ${ }^{4,9)}$ In this report, we described a patient with a distinct chondrodystrophy without ophthalmologic abnormality that is consistent with Kniestsyndrome.

\section{Case Report}

A 2-year-old female was referred to the genetics clinic for evaluation of her short stature and dysmorphic features. She showed a disproportionately short stature with a short and barrel-shaped chest and severe flexion deformities of the elbow, knee, and hipjoints.

She was born by $\mathrm{C}$-section due to cephalopelvic disproportion at 38 weeks of gestation (birth weight of $2.85 \mathrm{~kg}$ ) and had no definite perinatal asphyxia. She was the first only baby of her

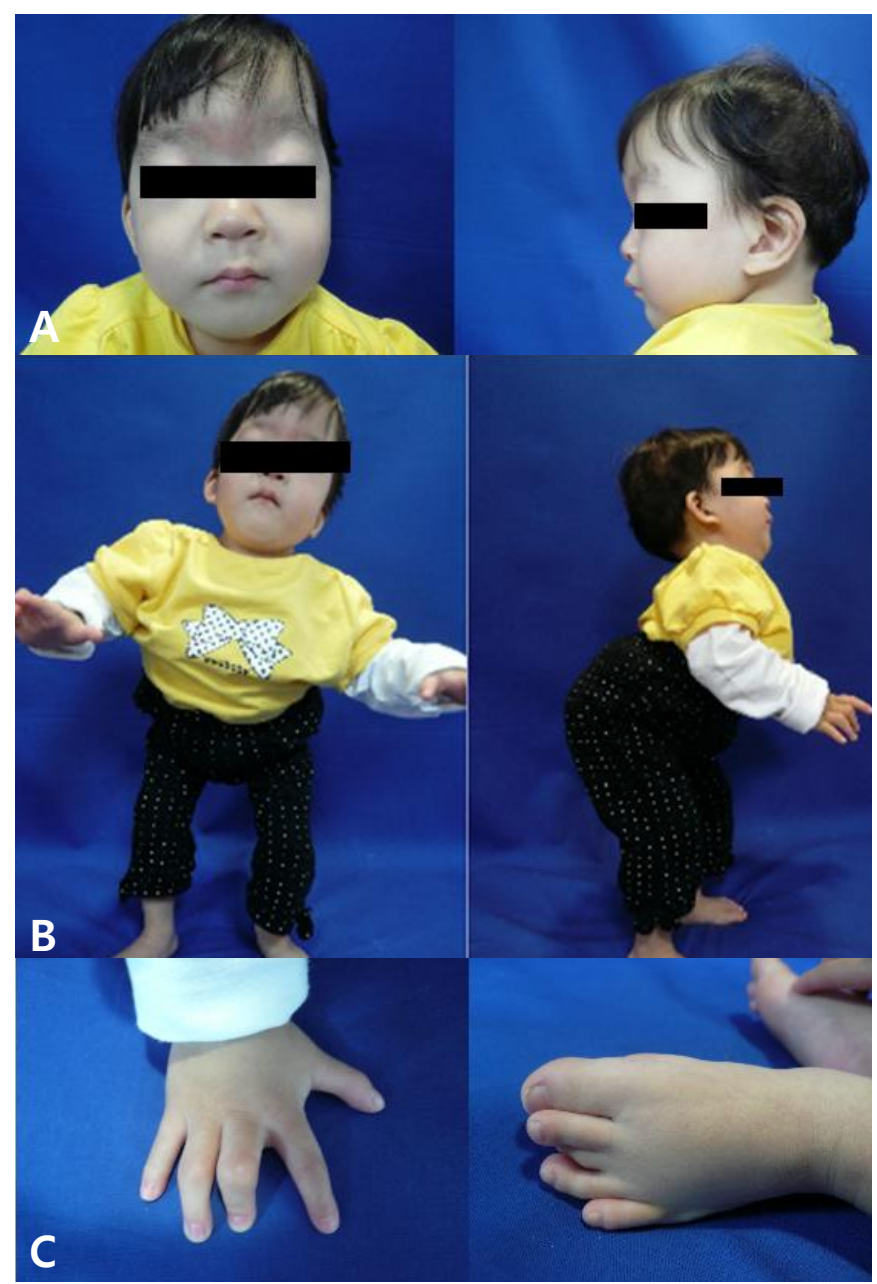

Fig. 1. Clinical features of the patient. Ptosis on both sides, with a short neck (A). Kyphoscoliosis and lordosis of the spine (B). Flexion deformities on multiple joints of the hands and feet (C). parents. Newborn examination revealed kyphosis with skin dimpling and nevus on the sacral area, which led to the diagnosis of spinal cord tethering and lipomeningomyelocele. Afterward, she underwent an operation for lipomeningomyelocele at 13 months of age.

On the initial physical examination, her height and weight were $77.3 \mathrm{~cm}$ and $9.4 \mathrm{~kg}$, both below the third percentile and her head circumference was $46 \mathrm{~cm}$ at the $50^{\text {th }}$ percentile, with normal developmental milestones and intelligence. The face was flat and round, with a low nasal bridge, short upturned nose, dolichocaphaly, short neck, and curved ear canals. Bilateral ptosis was observed, with clear corneas. She also showed shallow breathing due to her restrictive and barrel shaped chest and severe kyphoscoliosis in the lower back. The limbs were shortened and the large joints showed flexion deformity with limited mobility. Flexion contracture of both hips was marked and she showed a waddling gait with limited hip abduction (Fig. 1). The patient's $37-y e a r-o l d ~ m o t h e r ~ w a s ~ 140 \mathrm{~cm}$ in height and showed similar appearance to her daughter. She denied significant past medical history and had not undergone genetic evaluation for Kniest syndrome. The patient's father had normal height and no remarkable medical history.

Biochemical tests, including a coagulation assay, showed no abnormal findings. Proteinuria, microscopic hematuria, and pyuria were noted at the first examination, but subsequent urine tests were normal. Urinary GAG excretion test for mucopolysaccharides were normal. An abdominal ultrasonogram revealed no evidence of hydronephrosis or ureter dilatation.

Radiologic examinations showed abnormalities in the spine, ribs, long bones, and multiple joints. The main bony abnormalities were irregularity and flattening of the vertebral bodies, severe thoracic lordosis, lumbar kyphosis (Fig. 2A), and narrowed intercostal spaces. Flexion deformities were seen in multiple joints of the elbow, hip, knee, and interphalangeal joints. The epiphyses of distal femur and proximal tibia were enlarged (Fig 2. B-D). Developmental dysplasia of the hip was suspected based on the ultrasound examination. A spine MRI revealed various anomalies, such as fused lamina from $\mathrm{T} 1$ to $\mathrm{T} 4$ and $\mathrm{T} 5$ to $\mathrm{T} 10$, a hemivertebra at T6, and a split spine with syringomyelia. A follow-up spine MRI revealed a film terminale lipoma and the patient underwent detethering of the cord (Fig. 3). In addition, she has received regular fusion and growing rod insertions, following separation of the ribs and spine to expand the thoracic space.

Finally, she was diagnosed as a Kniest syndrome, based on radiological findings and clinical features, although direct 

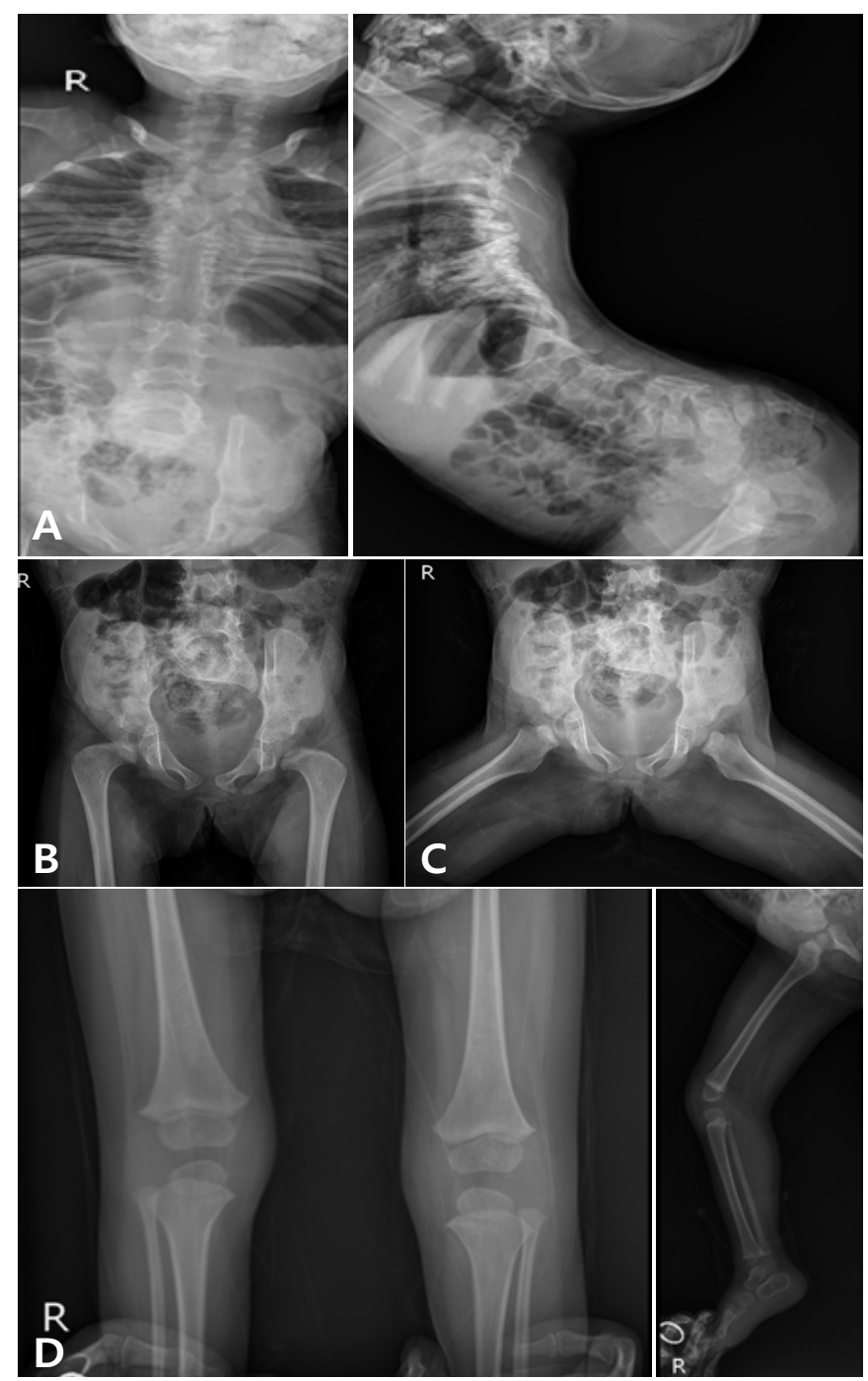

Fig. 2. Radiological findings of the patient. Irregularity and flattening of the vertebral bodies, severe thoracic lordosis, and lumbar kyphosis with intercostal space narrowing $(A)$. Flexion deformities in the hip joints, with hypoplastic femoral heads (B \& $C)$. Enlarged epiphyses of the femur and tibia (D). sequencing of $C O L 2 A 1$ was negative. The patient also received ophthalmologic examination which did not show abnormal findings, such as cataracts, lens dislocation, myopia, glaucoma, retinal detachment, and so on.

\section{Discussion}

Kniest syndrome (OMIM \#156550) is a rare skeletal dysplasia characterized by chondrodysplastic dwarfism, kyphoscoliosis, enlarged joints, visual loss, hearing loss, and cleft palate. ${ }^{4,10)}$ In 1952, Wilhelm described the original case of Kniest syndrome as a variant of metatropic dwarfism in a patient who showed short stature, restricted joint mobility, and blindness, but who was mentally alert and survived for a long time with an active life." Rimoin et al. recognized Kniest syndrome as a distinct entity ${ }^{2)}$ and Mortier et al. described a mutation in type II collagen gene that resulted in Kniest syndrome. ${ }^{10)}$ In Korea, the first case of Kniest syndrome was reported by Yoo YJ, et al in 1993. ${ }^{11)}$ Thereafter, there had been no further reports of Kniest syndrome in Korea.

The gene responsible for Kniest syndrome, $C O L 2 A 1$, is mapped to chromosome 12q13.11, which encodes three protein strands that make up alpha chain of type II collagen. Mutations of this gene interfere with the proper assembly of type II collagens and reduce the amount of these collagens. Collagens are complex molecules that provide structure, strength, and elasticity to connective tissue, such as the cartilage found in joints and the spinal column, the inner ear, and the vitreous humor. COL2A1 mutations can also cause several other skeletal disorders, including achondrogenesis type II/hypochondrogenesis (OMIM\#200610), Stickler dysplasia type I (OMIM\#108300), spondyloepiphyseal dysplasia
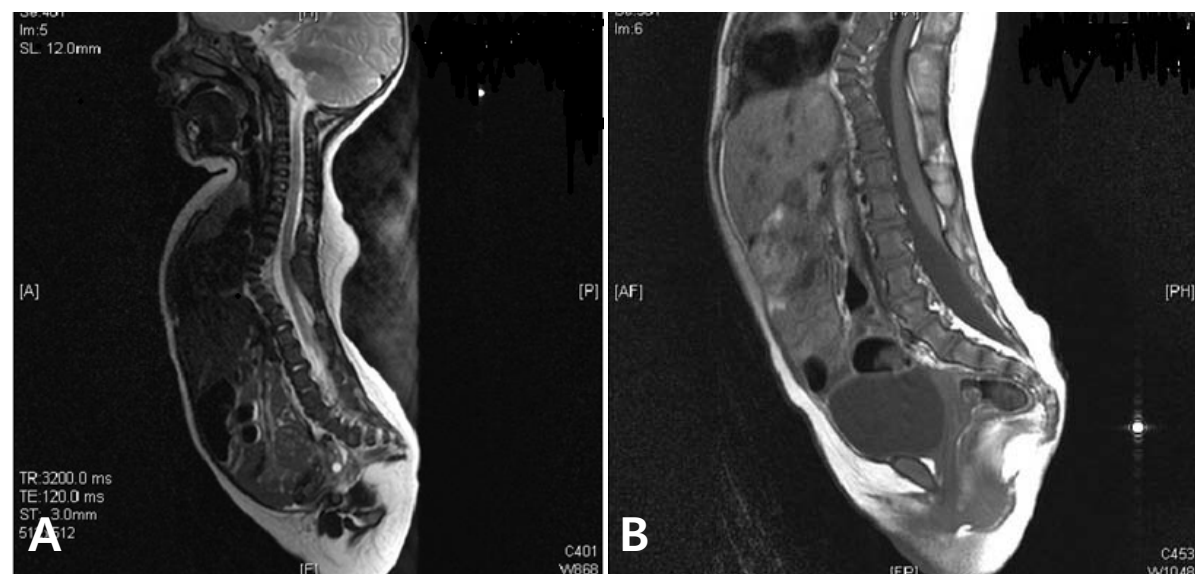

Fig. 3. Whole spine MRI shows fused lamina, from T1 to T4 and T5 to T10, aberrant focal lordotic curvature of the mid thoracic spine, and a split spine with syringomyelia $(A)$ and a film terminale lipoma (B). 
congenita (OMIM\#183900), spondyloepimetaphyseal dysplasia, Strudwick type (OMIM\#184250), spondyloperipheral dysplasia (OMIM\#271700), osteoarthritis with mild chondrodysplasia (OMIM\#604864), and platyspondylic lethal skeletal dysplasia, Torrance type (OMIM\# 151210). Most reported mutations of the COA2A1 gene in Kniest syndrome are small in-frame deletions within coding exons or splice site mutations that cause exon skipping. ${ }^{6,7)}$

The common phenotypic manifestations of the type II collagen disorders including high myopia, sensorineural hearing loss, cleft palate, and short trunked dwarfism, like as any mutation in COL2A1. Kniest syndrome should be differentiated from other forms of chondrodystrophies. Kniest syndrome usually shows moderate degree of phenotype among autosomal dominant type Il collagen disorder. Stickler syndrome and familial osteoarthritis show mild phenotypes in the disease spectrum, while spondyloepi (meta) physeal dysplasias (SED/SEMD) is in the middle and the lethal achondrogenesis type I//Hypochondrogenesis at the end of severe phenotype. Kniest syndrome, like SED congenita, can be phenotypically variable. ${ }^{12)}$

Radiographic findings are important for differential diagnosis of type II collargenopathies. Dumbbell-shaped femora and coronal clefts are distinct characteristics of Kniest syndrome, as is the "Swiss-cheese" appearance of the cartilage matrix. In Moriquio's disease, the pelvis shows supra-acetabular constriction, coxa valga, and narrow femoral necks. The lumbar vertebral bodies tend to be more severely affected than the thoracic. Characteristically, these patients have normal intelligence and could have minimal corneal clouding. They show flaring of the ribs, flared iliac wings, a steep acetabular roof, and proximal pointing of metacarpal bones. In SED, severe vertebral hypoplasia is notable at the thoraco-lumbar junction and the epiphysis is not bulbous. ${ }^{8)}$ Platyspondyly is more marked and the fibula is often longer in metatropic dwarfism than in Kniest syndrome. ${ }^{9)}$ Although platyspondyly and hypoplastic femoral head are seen in SED, SEMD and metatropic dwarfism usually have small-sized but normal appearing epiphyses. ${ }^{8)}$ Enlarged epiphyses with cloudlike calcification may only be seen in Kniest syndrome, ${ }^{13)}$ as those in our patient. Patients with Kniest and Morquio syndromes and SED all have rather flat faces, compared to the normal facial appearance in metatropic dwarfism.

Evaluation of hip, spinal and knee complications is necessary in Kniest syndrome. Hip replacement is sometimes warranted in adults. Additionally, an examination of the $\mathrm{C} 1$ and $\mathrm{C} 2$ vertebra should be performed to evaluate odontoid hypoplasia, if spinal fusion is needed. Regardless of whether a spinal fusion is required, lax ligaments in the neck could lead to spinal injury during anesthesia, contact sports, and car accidents. For this reason, a patient with Kniest syndrome should be cautioned to avoid activities/ sports that could result in trauma to the neck or head. In addition, the patient may have chest wall constriction, which can cause decreased lung capacity. Therefore, the patient should be closely monitored during anesthesia and for complications of respiratory infections. Anesthesia also presents risks related to cervical spine instability, lung capacity, and small airways and it should not be performed until these have been evaluated.

The first reported case of Kniest syndrome in Korea was a newborn with unknown birth and family history, who presented with severe cyanosis and respiratory difficulty, associated with cleft palate and had a disproportionate large head, a flat and round face, bilateral ptosis, a low and flat nasal bridge, barrelshaped chest, umbilical hernia, odontoid process, kyphoscoliosis and flexion contracture with dumbbell-shaped enlargement of epiphyses of long bones.11) Finally, this patient expired due to respiratory difficulty along with heart failure, on the ninth day of hospitalization. In Autopsy, the cartilage contained large chondrocytes intervening loose matrix, so called "Swiss cheese" pattern seen through light microscopy.11) Compared to this previously reported case, our case had similar phenotype, such as a flat and round face with a low nasal bridge, a short upturned nose, curved ear canals and bilateral ptosis, but had normal intelligence and no cleft palate. She had significant kyphosis and lordosis in conjunction with spinal cord tethering and a lipomeningomyelocele from birth.

Lipomeningomyelocele is not a common symptom and there has been no report of Kniest syndrome accompanying lipomeningomyelocele, yet. The patient has undergone spinal detethering and removal of lipomeningomyelocele. Every six months, she has undergone fusion and growing rod insertion following separation of ribs and spine to expand the thoracic space.

Regular eye examinations should be conducted by an ophthalmologist to evaluate for myopia and retinal detachment. Hearing should be checked and ear infections should be closely monitored. Tubes may need to be placed in the ears. Our case has received ophthalmologic exams which did not reveal abnormal findings. She has had no history of recurrent otitis media and was advised to undergo the evaluation of hearing.

Our case was diagnosed as a Kniest syndrome by clinical and radiographical findings, although the gene test was negative. Since Kniest syndrome is very rare and the test reliability or validity of DNA sequencing of COL2A1 mutations for Kniest syndrome have not yet been well described in the literature. In 1999, Wilkin 
et al. identified four dominant deletion mutations from exon 12 to 24 in COL2A1 in Kniest syndrome, all of which result in exon skipping, in addition to a previously known splice-site deletion. ${ }^{12)}$ In 2005, Nishimura et al. found 38 COL2A1 mutations in 41 out of 56 families suspicious of type II collagenopathies. Among these, all nine truncation or splice-site mutations in the triple helical or $\mathrm{N}$-propeptide region caused Stickler dysplasia type 1 or Kniest syndrome, and two new mutations (c.1023+2T4G and c.2304$1 \mathrm{G} 4 \mathrm{C}$ ) from these nine mutations were shown in patients with a Kniest syndrome phenotype. ${ }^{14)}$ Additional COL2A1 mutations may currently be under investigation. Therefore, further study may need to find other causative mutations or genes in Kniest syndrome.

\section{Acknowledgements}

The authors thank the clinical genetics laboratory at Severance Children's Hospital for assistance and the genetic work-up.

\section{References}

1. Kniest W. Differential diagnosis between dysostosis enchondralis and chondrodystrophy. Z Kinderheikd 1952;70:633-40.

2. Rimoin DL, Hollisster DW, Siggers $P$, Silberberg R, Lachman R, Mcalister $W_{1}$ et al. Clinical, radiographic, histologic and ultrastructural definition of the Kniest syndrome. Pediatr Res 1973;7:348.

3. Maumenee IH, Traboulsi El. The ocular findings in Kniest Dysplasia. Am J Ophthalmol 1985;100:155-60.
4. Jones. Smith's recognizable patterns of human malformation. 6th ed. Philadelphia: Elsevier Saunders Co., 1997;410-1.

5. Poole AR, Pidoux I, Reiner A, Rosenberg L, Hollister D, Murray L, et al. Kniest dysplasia is characterized by an apparent abnormal processing of the C-propeptide of type II cartilage collagen resulting in imperfect fibril assembly. J Clin Invest 1988;81:579-89.

6. Savarirayan R, Rimoin DL. The skeletal dysplasias. Best Pract Res Clin Endocrinol Metab 2002;16:547-60.

7. Spranger J, Winterpacht A, Zabel B. The Type II Collagenopathies: a spectrum of chondrodysplasias. Eur J Pediatr 1994;153:56-65.

8. Nagendran S, Richards, AJ, McNinch A, Sandford RN, Snead MP. Somatic Mosaicism and the Phenotypic Expression of COL2A1 Mutations. Am J Med Genet 2012;158:1204-7.

9. Kim HJ, Beatris NG, Brill P, Raab E, Hirschhorm K and Matalon R. Kniest Syndrome with Dominant Inheritance and Mucopolysacchariduria. Am J Hum Genet 1975;27:755-64.

10. Mortier GR, Wilkin DJ, Wilcox WR, Rimoin DL, Lachman RS, Ryre DR, et al. A radiographic, morphologic, biochemical and molecular analysis of a case of achondrogenesis type II resulting from substitution for a glycine residue (Gly691-to-Arg) in the type II collagen trimer. Hum Mol Genet 1995;4:285-8.

11. Yoo YJ, Na KC, Moon KR, Park SK, Park YB, Kee KH. A case of Kniest syndrome. J Korean Pediatr Soc 1993;36:138-43.

12. Wilkin DJ, Artz AS, South $S$, Lachman RS, Rimoin DL, Wilcox WR, et al. Small deletions in the type II collagen triple helix produce Kniest dysplasia. Am J Med Genet 1999;85:105-12.

13. Kozlowski K, Barylak A, Kobielowa Z. Kniest syndrome (report of two cases). Australas Radiol 1977;21:60-7.

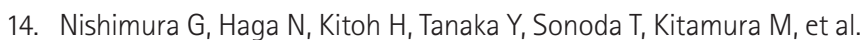
The phenotypic spectrum of COL2A1 mutations. Hum Mutat 2005; 26:36-43. 